\title{
In-Host Analysis of Malaria Dynamics in Humans
}

\author{
Kodwo Annan \\ School of Science \& Technology, Georgia Gwinnett College, Lawrenceville, USA \\ Email: kannan@ggc.edu
}

How to cite this paper: Annan, K. (2020) In-Host Analysis of Malaria Dynamics in Humans. Open Journal of Applied Sciences, 10, 191-209.

https://doi.org/10.4236/ojapps.2020.105015

Received: March 18, 2020

Accepted: May 10, 2020

Published: May 13, 2020

Copyright (c) 2020 by author(s) and Scientific Research Publishing Inc. This work is licensed under the Creative Commons Attribution International License (CC BY 4.0).

http://creativecommons.org/licenses/by/4.0/

\begin{abstract}
Human malaria infection poses a major global health threat worldwide. Yet, no sophisticated mathematical model exists to study the complex dynamics and interactions between the parasites and host immune response at the blood and liver stages. In this paper, an in-host mathematical model of Plasmodium falciparum malaria dynamics and interactions in an infected host cells are studied at the liver stage by incorporating the red blood cells and the immune system. Numerical simulations are applied to investigate the interactions between the host immune response, the parasite dynamics, and the disease dynamics at both the blood and liver stages. Results show that immunity has a significant impact in clearing infected red blood cells. Furthermore, the infected erythrocytes and hence the severity of malaria tend to increase with increasing density of merozoites in the blood. The result revealed that intervention during malaria infection should focus on minimizing merozoite invasion rate on healthy erythrocytes and the density of merozoites in circulation.
\end{abstract}

\section{Keywords}

Malaria, Red Blood Cells, Mathematical Modeling, Merozoite, Immune Cells

\section{Introduction}

Malaria is a multifactorial infectious disease that represents a serious public health problem especially in sub-Saharan Africa and Southeast Asia [1]. The disease is caused by parasites of the genus Plasmodium belonging to the apicomplexan phylum, which invades and reproduces in the erythrocytes. Hematophagous mosquitoes of the genus Anopheles are required for the transmission of the Plasmodium falciparum (the most virulent and prevalent parasite in Africa and Asia) invading humans and are responsible for most morbidity and mor- 
tality [2].

The main aspects of malaria infections can be summarized in three points: 1) the mechanism of transmission through the parasite life cycle between host and vector, 2) the clinical symptoms, showing illness, that depend on a specific stage of this life cycle and 3) the burden of morbidity and mortality [3]. In a single bite, an estimated average of 15 sporozoites is injected into the human body by a feeding mosquito infected with plasmodium falciparum [4] [5]. A sporozoite travels in the blood stream to the liver where it invades hepatic cells, matures into schizonts and produces 30,000 to 40,000 merozoites within 6 days. Each merozoite tries to invade a red blood cell where it reproduces asexually, and after approximately 48 hours the erythrocyte ruptures, releasing a number of merozoites to renew the cycle [6]. Some merozoites differentiate into gametocytes which are later picked up by a female anopheles mosquito during blood meal. The interaction between merozoites and red blood cells is well represented in the malaria parasite life cycle diagram in [7] [8]. The blood stage merozoite replication process is the main cause of malaria morbidity and mortality. The infection is associated with a reduction in haemoglobin levels, frequently leading to anaemia [9]. This happens especially when the destruction of red blood cells is followed by a decreased production of red blood cells from the bone marrow [7] [10].

Like most other infectious diseases, malaria infection triggers the immune system. The cells of the immune system are produced in the bone marrow and they include myeloid (neutrophils, basophils, eosinophils, macrophages and dendritic cells) and lymphoid (B lymphocyte, T lymphocyte and Natural Killer) cells [11] [12] [13] [14]. The myeloid induces red blood cells and platelets in addition to controlling the innate immune mechanism whilst lymphoid plays a crucial role in the adaptive immune response [13]. The T cells produced by the lymphoid undergo a process of maturation in the thymus differentiating into naive CD8+ cells and CD4+ T helper cells (Th1 and Th2). These helper cells later prime CD8+ cells to kill pathogens directly by cytotoxicity and indirectly by inducing B cells to produce antigen specific antibodies [14] [15] [16]. Immune response to malaria is greatly determined by the activities of antigen presenting cells (APCs), namely dendritic cells (DCs), macrophages and B lymphocytes. APCs capture and process antigens for presentation to T cells (CD4+ and CD8+ $\mathrm{T}$ cells) and it is reported in Annan et al. (2018). The contributions of the macrophages during phagocytosis and opsonization of malaria pathogens is well studied [15] [17] [18] [19] [20].

In addition, the dendritic cells recognize and capture malaria parasites and infected red blood cells at the Marginal Zone for presentation to CD4+ and $\mathrm{CD} 8+\mathrm{T}$ cells. This initiates differentiation of CD4+ $\mathrm{T}$ cells into $\mathrm{T}$ helper 1 (Th1), T helper 2 (Th2) cells and Regulatory $\mathrm{T}$ cells (Treg). Mature dendritic cells produce IL-12 inducing Th1 cell to produce inflammatory cytokine Interferon gamma (IFN- $\gamma$ ), which makes CD8+ T cells become cytotoxic, hence hav- 
ing the capacity to phagocytose. IL-12 activates natural Killer (NK) cells, which further enhances the production of (IFN- $\gamma$ ) [17] [20]. Macrophages activated by $($ IFN $-\gamma$ ) produce inflammatory cytokines which regulate intercellular adhesion molecule 1 (ICAM-1) [21], and help bind infected erythrocytes in patients with severe malaria diagnosis [21] [22]. The binding of uninfected and parasite infected erythrocytes to endothelial cells has been examined in vitro by [23] with the result of binding of parasite infected erythrocytes being significantly higher than that of uninfected erythrocytes. This result may be due to the fact that uninfected erythrocyte binding is independent of IFN- $\gamma$.

Furthermore, through phagocytosis, macrophages and dendritic cells becoming heavily loaded with haemozoins due to uptake of hemoglobin, their functionality is impeded as antigen-presenting cells and large production of pro-inflammatory cytokines, (IFN- $\gamma$ ) and (TNF- $\alpha$ ) are created [23]. However, these unpleasant inflammatory responses do not continue indefinitely as they are often regulated by some immunosuppressive anti-inflammatory cytokines such as the immunomodulatory cytokines IL-10 and Transforming growth factor $\beta$ (TGF- $\beta$ ), thus, limiting the pathology of disease [23] [24] [25]. High ratios of (IFN- $\gamma$ ), (TNF- $\alpha$ ) and IL-12 to TGF- $\beta$ or IL-10 are associated with decreased risk of malaria infection but increase risk of those who actually become infected [25]. Since malaria pathogenesis in the human host is very complex, many epidemiological researchers have used mathematical models to describe in-host interaction of blood stage malaria parasites with red blood cells and the immune system [26] [27] [28]. This is because the merozoite and the gametocyte formation stages occur in the human host and the blood stage merozoite replication process is the main cause of the diseases' morbidity and mortality.

Malaria parasite interaction with the human host was first modeled mathematically by Anderson et al. [29] [30] where they examined the malaria parasite life cycle suggested that the effectiveness of the immunological response was inversely proportional to the life expectancy of the target stage of the parasite development cycle. The model was adjusted by Hetzel et al. to investigate blood-stage malaria infection [31]. In the new model, merozoites were assumed to be absorbed into already infected erythrocytes, however, they did not include the immune system. They observed that the rates creation and death of merozoite and erythrocyte were favored by the persistent invasion of the red blood cells. Hence suggested that without immunity, the density of susceptible erythrocytes in the red blood cells were crucial in determining the initial pattern of malaria infection. Analysis of the model with simple immune response resulted in effective destruction of infected red blood cells by controlling the parasite density.

Recently, several studies on mathematical modeling of in-host malaria and its dynamics within the human host have been done. Nearly all the earlier mathematical models focused on improving Plasmodium falciparum control while focusing on the blood stage of parasite development [32] [33] [34] [35]. 
These models have been found to be useful in explaining in-host observations such as parasite diversity [36]; predicting the impact of interventions or the use of antimalarial [37]; dynamics of densities of healthy erythrocytes, infected erythrocytes and free merozoites [38]; age-structured model [39]; in-host model with immune response to regulate malaria parasites [40] [41] [42] [43]. Although models [40] [41] [42] [43] considered the impact of immune response and treatment, models in [41] [42] [43] were limited only to the blood stage of Plasmodium falciparum development. Chiyaka et al. [40] came up with an improved model that incorporated immune factors, red blood cells, infected red blood cells, merozoites, B cells, and antibodies. However, their model did not account for the natural death rate of infected red blood cells that may occur within the period of schizogony. Tuwiine et al. [44] improved on this limitation, but omitted an important biological interaction of the innate response and the pathogen. Thus, none of the in-host models show explicitly the effect of innate immune response on the pathology of the disease or account for the natural death rate of infected red blood cells during the period of schizogony. In [45] [46] [47], the liver stage is incorporated into the malaria model. However, the contribution of immune system was ignored. Moreover, all the immune cells were assumed to play an active role during malaria infection in [46] [47]. This may not be entirely true. The specific impacts of immune responses to malaria infection are well discussed in [48] and in other earlier papers [49] [50] [51] [52].

In this paper, we extended the model in [40] by incorporating the liver stage of parasite development and adapted the immune response discussed in [48]. The reformulated in-host malaria model focused on the post-liver stage and described the dynamics of interactions between the malaria parasites, infiltration of the red blood cells by merozoites. We also examined the immune system cells activation that aimed to interfere and destroy the malaria infection. The understanding of the dynamics of the parasite at the post-liver stage in human host is crucial to the quest for malaria elimination and eradication.

We formulate the in-host malaria model equations, dimensionless the system of equations, and perform model parametrization in the next section. Section 3 is devoted to numerical simulations of the in-host model interactions with malaria parasites, red blood cells, and immunity effectors. We then give some discussion and conclusion of the paper in Section 4.

\section{Model Formulation}

\subsection{Variables Description \& Equations}

The health of the susceptible individual depends on the red blood cell count and is denoted as density $C_{r}$. Let the concentration of merozoite released from the liver at $t=0$ be $C_{m}$ and the concentration of infected red blood cells by merozoites be $I_{r}$. The maturation of infected red blood cells results in the production of subsequent generations of merozoites with some forming asexual game- 
tocytes denoted by $C_{g}$. For simplicity, we represent the blood-borne phagocytic inmate immune cells (NK, macrophages and dendritic cells) concentration by $C_{i}$. Antibodies produced in large amount by B cells induced through T-cells activation in response to signals from $C_{i}$ is denoted by $C_{a}$.

Since the presence of antibodies restrict the infection of the red blood cells by the merozoites, we state the infection rate of red blood cells by merozoites as $\frac{\beta_{C r} C_{r} C_{m}}{1+c_{o} C_{a}}$, where $\beta_{C r}$ is the infection rate of red blood cells, $c_{0}$ the efficiency of antibodies hindering merozoite release, and the factor $\frac{1}{1+c_{o} C_{a}}$ denoting antibodies in blocking and reducing erythrocytic invasion. Since red blood cells develop continuously from stem cells in the bone marrow through reticulocytes within seven days and can live up to 120 days [39], we assume a rate of fresh erythrocytes conscription $\lambda_{C r}$ from the bone marrow and a natural death rate of $\mu_{C r} C_{r}$. Thus, the equation for the infected red blood cells is:

$$
\frac{\mathrm{d} C_{r}}{\mathrm{~d} t}=\lambda_{C r}-\frac{\beta_{C r} C_{r} C_{m}}{1+c_{0} C_{a}}-\mu_{C r} C_{r} .
$$

The removal of infected red blood cells by the innate immune cells through phagocytosis is given by $k_{I r} C_{i} I_{r}\left(1+k_{C a} C_{a}\right)$, where $k_{I r}$ is the elimination rate of infected red blood cells by innate immune cells, $k_{C a}$ is the antibodies induced Fc-dependent killing rate of infected red blood cells. Assuming that in the first two days of parasitized erythrocyte schizogony the infected red blood cells die at rate $\mu_{n} I_{r}$ or survive to experience a per capita death rate due to parasite rupture of $\mu_{I r}$. With no loss of blood cells on infection, $\left(\mu_{I r}+\mu_{n}\right) I_{r}$, we have

$$
\frac{\mathrm{d} I_{r}}{\mathrm{~d} t}=-\left(\mu_{I r}+\mu_{n}\right) I_{r}+\frac{\beta_{C r} C_{r} C_{m}}{1+c_{0} C_{a}}-k_{I r} C_{i} I_{r}\left(1+k_{C a} C_{a}\right),
$$

If the number of merozoites per busting schizont, on average, is $x$ merozoites and the fraction of merozoites committed to gametocytogenesis is $\varepsilon$, then the net rate of merozoites production by the infected red blood cells is $\frac{x \mu_{I r} I_{r}(1-\varepsilon)}{1+c_{1} C_{a}}$, where $c_{1}$ is the efficiency of antibodies preventing the release of new merozoites. Assume that antibodies assist phagocytic cells in killing merozoites and that they inhibit gametocyte development in the host by binding to young gametocytes during their developmental stages. The rate of merozoites and gametocytes killing through binding can be expressed as $k_{m} k_{b} C_{m} C_{a} C_{i}$ and $k_{g} k_{c} C_{g} C_{a} C_{i}$, respectively, where $k_{m}$ is the elimination rate of merozoites by innate immune cells and $k_{g}$ is the elimination rate of gametocytes by innate immune cells. Thus, if we represent the natural death rates or portion killed by innate and adaptive immune cells of gametocytes and merozoites by $\mu_{g} C_{g}$ and $\mu_{m} C_{m}$, then we have the merozoites and gametocytes equations as,

$$
\frac{\mathrm{d} C_{m}}{\mathrm{~d} t}=\frac{x \mu_{I r} I_{r}(1-\varepsilon)}{1+c_{1} C_{a}}-\frac{\beta_{C r} C_{r} C_{m}}{1+c_{0} C_{a}}-\mu_{m} C_{m}-k_{m} C_{i} C_{m}\left(1+k_{b} C_{a}\right) \text {, and }
$$




$$
\frac{\mathrm{d} C_{g}}{\mathrm{~d} t}=\frac{x \mu_{I r} I_{r} \varepsilon}{1+c_{1} C_{a}}-\mu_{g} C_{g}-k_{g} C_{i} C_{g}\left(1+k_{c} C_{a}\right) .
$$

Assume that immune cells coming from the bone marrow's stem cells have rate $b_{m}$ and the one stimulated by the presence of infected red blood cells and merozoites have a per capita rate, with an induced rate of innate immune cell production of $\eta_{1}\left(I_{r}+\phi C_{m}\right)$ where $\phi$ indicates the phagocyte growth difference between merozoites and infected red blood cells. Since malaria specific antibodies are associated with merozoites and sporoziotes, we write the rate of antibody's enrolment of merozoites and malaria parasite as $\eta_{2}\left(I_{r}+g_{2} C_{m}\right)\left(t-d_{1}\right)$. Here, $g_{1}$ is the adaptive immunity difference between merozoites and infected red blood cells, and $d_{1}$ is the time delay between parasites contact and the production of the adaptive cells. The deteriorating rates of innate immune cells and antibodies interacting with the parasites are represented as $C_{i}\left(k_{I r} I_{r}+k_{m} C_{m}\right)$ and $C_{a}\left(\eta_{3} I_{r}+\eta_{4} C_{m}\right)$, respectively. Furthermore, assuming that antibodies die at a rate $\mu_{t}\left(C_{a_{0}}-C_{a}\right)$, where $C_{a_{0}}$ is the initial antibodies density. Thus, the innate immune cells and antibodies equations are formulated as:

$$
\begin{gathered}
\frac{\mathrm{d} C_{i}}{\mathrm{~d} t}=b_{m}+\eta_{1}\left(I_{r}+\phi C_{m}\right)-\mu_{i} C_{i}-C_{i}\left(k_{d} C_{r}+k_{n} C_{m}\right) \text {, and } \\
\frac{\mathrm{d} C_{a}}{\mathrm{~d} t}=\eta_{2}\left(I_{r}+g_{2} C_{m}\right)\left(t-d_{1}\right)+\mu_{t}\left(C_{a_{0}}-C_{a}\right)-C_{a}\left(\eta_{3} I_{r}+\eta_{4} C_{m}\right) .
\end{gathered}
$$

\subsection{Initial Conditions}

The initial condition for $C_{r}$ is the concentration of red blood cells of an uninfected individual which is represented by the steady state rate $\frac{\lambda_{r}}{\mu_{r}}$. Similarly, the initial steady state rate of the innate immune cells for an individual is $\frac{b_{m}}{\mu_{i}}$. Annan et al. (2017) predicted that for a successful infectious bite of a mosquito, an average of 525,000 merozoites is produced and thus we estimate the initial concentration of merozoites released into the blood stream as 0.105 cells per $\mu_{l}$. The concentrations of infected red blood cells and gametocytes are estimated as zero at the release of hepatic merozoite, since it is the blood stage merozoites that penetrate red blood cells and eventually convert to gametocytes. Furthermore, the choice of $C_{a_{0}}$ as a non-zero initial density of antibodies is important because instead of restricting the model to only a single contact it makes provision for subsequent contacts which lead to accumulated immunity from previous infections. At initial contact, $C_{a_{0}}=0$ and since the number of memory cells does not change much over the course of a single infection, $C_{a_{0}} \rightarrow 0$ as the parasites are cleared. Nevertheless, for an additional infection before antibodies completely decline to zero, we have $C_{a} \rightarrow C_{a_{0}}$. Thus, the system's initial conditions are:

$$
C_{r}(0)=\frac{\lambda_{r}}{\mu_{r}}, I_{r}(0)=0, C_{m}(0)=0.105, C_{g}(0)=0, C_{i}(0)=\frac{b_{m}}{\mu_{i}}, C_{a}(0)=0 .
$$


Since Equation (6) is dependent on the solution at the previous time $t-d_{1}$, it is necessary to state an initial functions to specify the solution before time $t=0$ as

$$
C_{r}(t)=\frac{\lambda_{r}}{\mu_{r}}, I_{r}(t)=0, C_{m}(t)=0.105, C_{g}(t)=0, C_{i}(t)=\frac{b_{m}}{\mu_{i}}, C_{a}(t)=0, \forall t<0 .
$$

In addition, the system of equation works for a number of time scales derived in [20] [43] [48] that include natural red blood cell turnover ( $\sim 120$ days), blood infiltrated merozoite maturation in blood cells ( $\sim 2$ days), non-infiltrated merozoite survival ( $\sim 30$ minutes), and adaptive immune response ( $\sim 2$ days).

\subsection{Dimensionless Model}

We scale time with the maturation of merozoites and state $t=\frac{\hat{t}}{\mu_{r}}$, where $\frac{1}{\mu_{r}}$ is the duration of schizogony leading to cell induced death of infected red blood cells and subsequent release of new generation merozoites. The dimensionless time, $\hat{t}=1$ is a time scale of about 2 days and we note here that it is relatively longer time compared with the longevity of merozoites and their invasion capacity. The choice of this scaling enables the study of the model in relation to the replication rate of blood stage parasites. The red blood cell equation is normalized with the steady state rate $\frac{\lambda_{r}}{\mu_{r}}$. The infected red blood cells, merozoites and gametocyte are rescaled using the same parameter. Also, the innate immune density is rescaled using $\frac{b_{m}}{\mu_{i}}$ and antibodies with activation rescaled by $\frac{1}{c_{0}}$.

Introducing new variables in terms of proportion as follows:

$$
C_{r}=\frac{\lambda_{r}}{\mu_{r}} C_{r}^{*}, I_{r}=\frac{\lambda_{r}}{\mu_{r}} I_{r}^{*}, C_{m}=\frac{\lambda_{r}}{\mu_{r}} C_{m}^{*}, C_{g}=\frac{\lambda_{r}}{\mu_{r}} C_{g}^{*}, C_{i}=\frac{b_{m}}{\mu_{i}} C_{i}^{*}, C_{a}=\frac{1}{C_{0}} C_{a}^{*} .
$$

Thus, we define the dimensionless parameters below with their parametric values defined in Table 1.

$$
\begin{aligned}
& \beta=\frac{\beta_{r} \lambda_{r}}{\mu_{r} \mu_{I}}, b=\frac{\mu_{i}}{\mu_{I}}, d=\frac{\mu_{m}}{\mu_{I}}, \sigma=\frac{\mu_{r}}{\mu_{I}}, \mu=\frac{\mu_{n}}{\mu_{I}}, k_{1}=\frac{k_{a}}{c_{0}}, k_{2}=\frac{k_{b}}{c_{0}}, k_{3}=\frac{k_{c}}{c_{0}}, \\
& k=\frac{k_{I} b_{m}}{\mu_{i} \mu_{I}}, e=\frac{\mu_{g}}{\mu_{I}}, \alpha=\frac{k_{m} b_{m}}{\mu_{i} \mu_{I}}, f=\frac{k_{g} b_{m}}{\mu_{i} \mu_{I}}, \omega=\frac{\lambda_{r} \mu_{i} \eta_{1}}{b_{m} \mu_{i} \mu_{I}}, h_{1}=\frac{\lambda_{r} \eta_{3}}{\mu_{i} \mu_{I}}, h_{2}=\frac{\lambda_{r} \eta_{4}}{\mu_{i} \mu_{I}}, \\
& \phi=c_{0} C_{a}, \rho_{1}=\frac{c_{0} \lambda_{r}}{\mu_{i} \mu_{I}}, \rho_{3}=\frac{\mu_{a}}{\mu_{i}}, k_{4}=\frac{k_{d} \lambda_{I}}{\mu_{i} \mu_{I}}, k_{5}=\frac{k_{n} \lambda_{I}}{\mu_{i} \mu_{I}}, \tau=d_{1} \mu_{i}, k_{6}=\frac{c_{1}}{c_{0}} .
\end{aligned}
$$

Consequently, the dimensionless model Equations (1)-(6) becomes:

$$
\begin{gathered}
\frac{\mathrm{d} C_{r}}{\mathrm{~d} t}=\sigma\left(1-C_{r}\right)-\frac{\beta C_{r} C_{m}}{1+C_{a}}, \\
\frac{\mathrm{d} I_{r}}{\mathrm{~d} t}=\frac{\beta C_{r} C_{m}}{1+C_{a}}-(1-\mu) I_{r}-k C_{i} I_{r}\left(1+k_{1} C_{a}\right), \\
\frac{\mathrm{d} C_{m}}{\mathrm{~d} t}=\frac{x_{i}(1-\varepsilon) I_{r}}{1+k_{6} C_{a}}-\frac{\beta C_{r} C_{m}}{1+C_{a}}-d C_{m}-\alpha C_{i} C_{m}\left(1+k_{2} C_{a}\right),
\end{gathered}
$$




$$
\begin{gathered}
\frac{\mathrm{d} C_{g}}{\mathrm{~d} t}=\frac{\varepsilon X_{i} I_{r}}{1+k_{6} C_{a}}-e C_{g}-f C_{i} C_{g}\left(1+k_{3} C_{a}\right), \\
\frac{\mathrm{d} C_{i}}{\mathrm{~d} t}=b\left(1-C_{i}\right)+\omega\left(I_{r}+\phi C_{m}\right)-C_{i}\left(k_{4} I_{r}+k_{5} C_{m}\right), \\
\frac{\mathrm{d} C_{a}}{\mathrm{~d} t}=\rho_{1}(t-\tau)\left(I_{r}+\rho_{2} C_{m}\right)+\rho_{3}\left(\phi_{0}-C_{a}\right)-C_{a}\left(h_{1} I_{r}+h_{2} C_{m}\right),
\end{gathered}
$$

Subject to the history and initial conditions below:

$$
\begin{aligned}
& {\left[C_{r}(t), I_{r}(t), C_{m}(t), C_{g}(t), C_{i}(t), C_{a}(t)\right]} \\
& = \begin{cases}(1,0,0,0,1,0) & \text { for } t<0 \\
\left(1,0,1.05 \times 10^{-7}, 0,1,0\right) & \text { for } t=0\end{cases}
\end{aligned}
$$

\subsection{Model Parameterization}

Equations (7)-(12) are solved using the dimensionless parameter values defined in Table 1. The values in Table 1 are expressed in terms of $\kappa \ll 1$, a small parameter, to allow the investigation of timescale asymptotic analyses of the parasites interactions with the host's red blood cells. In this study, $\kappa$ describes a state where the death rate of merozoites is larger than the death rate of infected red blood cells. Thus, triggering merozoite survival a direct consequence of parasite replication and disease pathology.

Dimensional parameter values stated in Table 2 are either estimated from experimental/mathematical model sources or are taken from peer reviewed publications. Furthermore, we determine the remaining dimensional values using the combinations of parameters expressed in Table 1.

\section{Numerical Simulations}

Numerical simulations describing the events of in-host model were presented in Figures 1-5. The model had a single constant delay, $x$ in the antibodies concentration equation that expressed the time lag before the activation of adaptive immune response. The system of the delay differential equations was numerically solved using MATLAB with the parameter values summarized in Table $1 \& \mathrm{Ta}$ ble 2 and initial conditions as follows: $C_{r}=1, I_{r}=0, C_{m}=1.05 \times 10^{-7}, C_{g}=C_{a}=0$ and $C_{i}=1$, derived in Annan et al., [20]. These conditions described a situation where a susceptible human (that is $C_{a}(0)=\phi_{0}$ ), is exposed to malaria infection for the first time.. Here, a hepatic merozoites from a single infectious bite were released into the blood stream to undergo the first phase of erythrocytic schizogony. The individual having normal concentration of red blood cells $\left(C_{r}=1\right)$ and normal innate immune cell concentration $\left(C_{i}=1\right)$ was infected with an initial concentration of merozoites $\left(C_{m}=1.05 \times 10^{-7}\right.$ representing 35,000 merozoites [51]). The program was run in MATLAB using a relative and absolute tolerance of $10^{-7}$ with a delay of two days. The history conditions were the same as the initial conditions for $t \geq 0$.

In Figure 1, merozoites invade red blood cells and the immune cells respond 
Table 1. Dimensionless parameter values of in-host model in terms of a small parameter $\kappa=\frac{\mu_{n}}{\mu_{m}} \approx 0.001$

\begin{tabular}{|c|c|c|c|}
\hline Dimensional & Dimensionless & Value & Value in $k$ \\
\hline$\mu_{r} / \mu_{I}$ & $\sigma$ & 0.02 & $\kappa$ \\
\hline$\beta_{r} \lambda_{r} / \mu_{r} \mu_{I}$ & $\beta$ & 49 & $\mathrm{O}\left(\kappa^{-1}\right)$ \\
\hline$\mu_{i} / \mu_{I}$ & $b$ & 0.6 & $\mathrm{O}(1)$ \\
\hline$\mu_{m} / \mu_{I}$ & $d$ & 96 & $\mathrm{O}\left(\kappa^{-1}\right)$ \\
\hline$\mu_{n} / \mu_{I}$ & $\mu$ & 0.11 & $\mathrm{O}(1)$ \\
\hline$\mu_{g} / \mu_{I}$ & $e$ & 0.034 & $\mathrm{O}(\kappa)$ \\
\hline$k_{g} b_{m} / \mu_{i} \mu_{I}$ & $f$ & 0.35 & $\mathrm{O}(1)$ \\
\hline$\lambda_{r} \eta_{3} / \mu_{i} \mu_{I}$ & $h_{1}$ & 0.45 & $\mathrm{O}(1)$ \\
\hline$\lambda_{r} \eta_{4} / \mu_{i} \mu_{I}$ & $h_{2}$ & 0.34 & $\mathrm{O}(1)$ \\
\hline$k_{I} b_{m} / \mu_{i} \mu_{I}$ & $k$ & 0.23 & $\mathrm{O}(1)$ \\
\hline$k_{a} / c_{0}$ & $k_{1}$ & 2.3 & $\mathrm{O}(1)$ \\
\hline$k_{b} / c_{0}$ & $k_{2}$ & 20 & $\mathrm{O}(1)$ \\
\hline$k_{c} / c_{0}$ & $k_{3}$ & 2.1 & $\mathrm{O}(1)$ \\
\hline$k_{d} \lambda_{I} / \mu_{i} \mu_{I}$ & $k_{4}$ & 0.028 & $\mathrm{O}(\kappa)$ \\
\hline$k_{n} \lambda_{I} / \mu_{i} \mu_{I}$ & $k_{5}$ & 0.031 & $\mathrm{O}(\kappa)$ \\
\hline$c_{1} / c_{0}$ & $k_{6}$ & 15.2 & $\mathrm{O}(1)$ \\
\hline$k_{m} b_{m} / \mu_{i} \mu_{I}$ & $\alpha$ & 30 & $\mathrm{O}\left(\kappa^{-1}\right)$ \\
\hline$\lambda_{r} \mu_{i} \eta_{1} / b_{m} \mu_{i} \mu_{I}$ & $\omega$ & 10 & $\mathrm{O}(1)$ \\
\hline$c_{0} \lambda_{r} / \mu_{i} \mu_{I}$ & $\rho_{1}$ & 1.8 & $\mathrm{O}(1)$ \\
\hline$\rho_{2}$ & $\rho_{2}$ & 1.86 & $\mathrm{O}(1)$ \\
\hline$\mu_{a} / \mu_{i}$ & $\rho_{3}$ & 0.6 & $\mathrm{O}(1)$ \\
\hline$C_{0} C_{a}$ & $\phi$ & 2 & $\mathrm{O}(1)$ \\
\hline$m_{0}$ & $m_{0}$ & $1.05 \times 10^{-7}$ & $\mathrm{O}\left(\kappa^{2}\right)$ \\
\hline$x_{i}$ & $x_{i}$ & 16 & $\mathrm{O}(1)$ \\
\hline$\varepsilon$ & $\varepsilon$ & 0.0064 & $\mathrm{O}(\kappa)$ \\
\hline
\end{tabular}

by attacking and eradicating pathogens, leading to red blood cell recovery and subsequent drop down of immune cells.

Specifically, the blue dots in Figure 1(a) represent data on the red blood cells concentration of a malaria patient. The data were extracted from a data set obtained from the South Carolina State hospitals in which prior uninfected neurosyphilis patients were inoculated with P. falciparum malaria parasites as a means of malaria therapy for neurosyphilis. The infected red blood cells concentration dropped to a minimum level due to the merozoites invasion and then increased at the time when the immune system gained control. The lowest red blood cells concentration level of 0.55 occurred around $t=5.4$ days. When the red blood 
Table 2. In-host model dimensional parameter values. Values with asterisk $\left(^{*}\right)$ are mathematical model estimates and values with bullet $(\bullet)$ are obtained from experimental sources.

\begin{tabular}{|c|c|c|c|}
\hline Dimensional & Value & Unit & Source \\
\hline$\lambda_{r}$ & $4.15 \times 10^{4} *$ & cell/ $\mu \mathrm{l} /$ day & \\
\hline$\beta_{r}$ & $4.9 \times 10^{-6}$ & $\mu \mathrm{l} /$ cell/day & estimated \\
\hline$\mu_{r}$ & $8.3 \times 10^{-3} \bullet$ & /day & \\
\hline$\mu_{n}$ & $5.5 \times 10^{-2}$ & /day & estimated \\
\hline$\mu_{I}$ & $0.5 \bullet$ & /day & \\
\hline$\mu_{m}$ & $48 \bullet$ & /day & \\
\hline$\mu_{g}$ & $2.0 \times 10^{-2}$ & /day & estimated \\
\hline$c_{0}$ & $0.6 *$ & cell $/ \mathrm{mol}$ & \\
\hline$c_{1}$ & 1.12 & cell $/ \mathrm{mol}$ & estimated \\
\hline$k_{a}$ & 1.38 & cell $/ \mathrm{mol}$ & estimated \\
\hline$k_{b}$ & 12 & cell $/ \mathrm{mol}$ & estimated \\
\hline$k_{c}$ & 1.26 & cell $/ \mathrm{mol}$ & estimated \\
\hline$k_{r}$ & $0.9 *$ & $\mu \mathrm{l} /$ cell/day & \\
\hline$k_{m}$ & $1.18 \times 10^{2}$ & $\mu \mathrm{l} /$ cell/day & estimated \\
\hline$k_{g}$ & 1.4 & $\mu \mathrm{l} /$ cell/day & estimated \\
\hline$b_{m}$ & $3.8 \times 10^{-2}$ & cell/ $\mu \mathrm{l} /$ day & estimated \\
\hline$\eta_{1}$ & $1.3 \times 10^{-7}$ & /day & estimated \\
\hline$\eta_{2}$ & $3.0 \times 10^{-7}$ & $\mathrm{~mol} \mu \mathrm{l} / \mathrm{cell}^{2} /$ day & estimated \\
\hline$\eta_{3}$ & $4.5 \times 10^{-8}$ & $\mu \mathrm{l} /$ cell/day & estimated \\
\hline$\eta_{4}$ & $3.4 \times 10^{-8}$ & $\mu \mathrm{l} /$ cell/day & estimated \\
\hline$k_{d}$ & $\rho_{1}$ & 1.8 & $\mathrm{O}(1)$ \\
\hline$k_{n}$ & $\rho_{2}$ & 1.86 & $\mathrm{O}(1)$ \\
\hline$\mu_{p}$ & $\rho_{3}$ & 0.6 & $\mathrm{O}(1)$ \\
\hline$C_{0}$ & $\phi$ & 2 & $\mathrm{O}(1)$ \\
\hline$\mu_{c}$ & $m_{0}$ & $1.05 \times 10^{-7}$ & $\mathrm{O}\left(\kappa^{2}\right)$ \\
\hline
\end{tabular}

cells concentration reached the minimum level, the concentrations of infected red blood cells, merozoites, and gametocytes all peaked with similar shapes indicating some form of proportionality, as shown in Figures 1(b)-(d). The observations were expected since merozoites were expected to convert to infected red blood cells after a successful penetration.

The innate immune cells graph depicted in Figure 2 described how innate immune cells reacted to malaria pathogens in the host by proliferating to a peak value and then leveling off. During the beginning of the infection, the innate immune cells concentration preserved its normalcy and just after the concentration of the parasites attained its maximum level that the red blood cells concentration reached its minimum. This resulted in rapid increase in the adaptive 


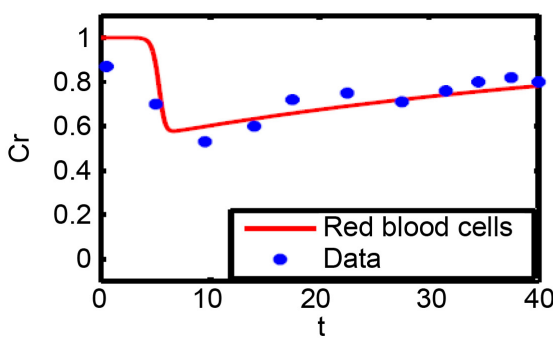

(a)

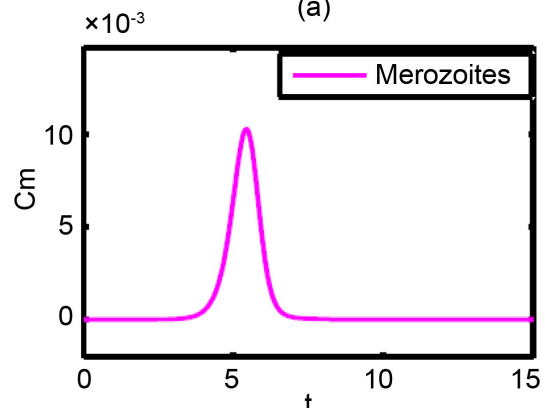

(c)

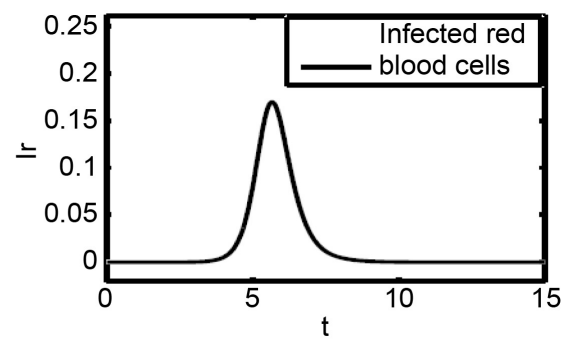

(b)

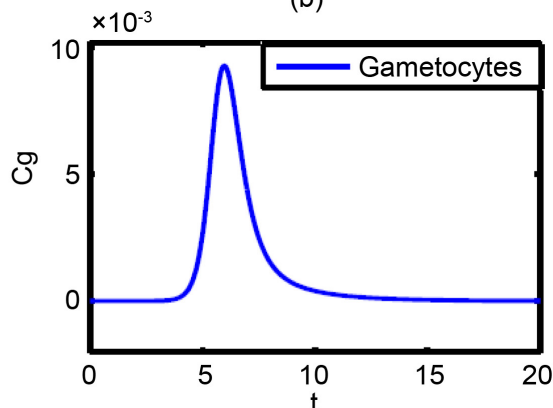

(d)

Figure 1. The figure shows the pathogenesis of malaria in human host with initial introduction of merozoites invading red blood cells. In (a), the blue dots represent data of strain malaria infected patient. Time equals to one in $1 b$ represents 2 days in real time and it corresponds to the period of each erythrocytic schizogony.
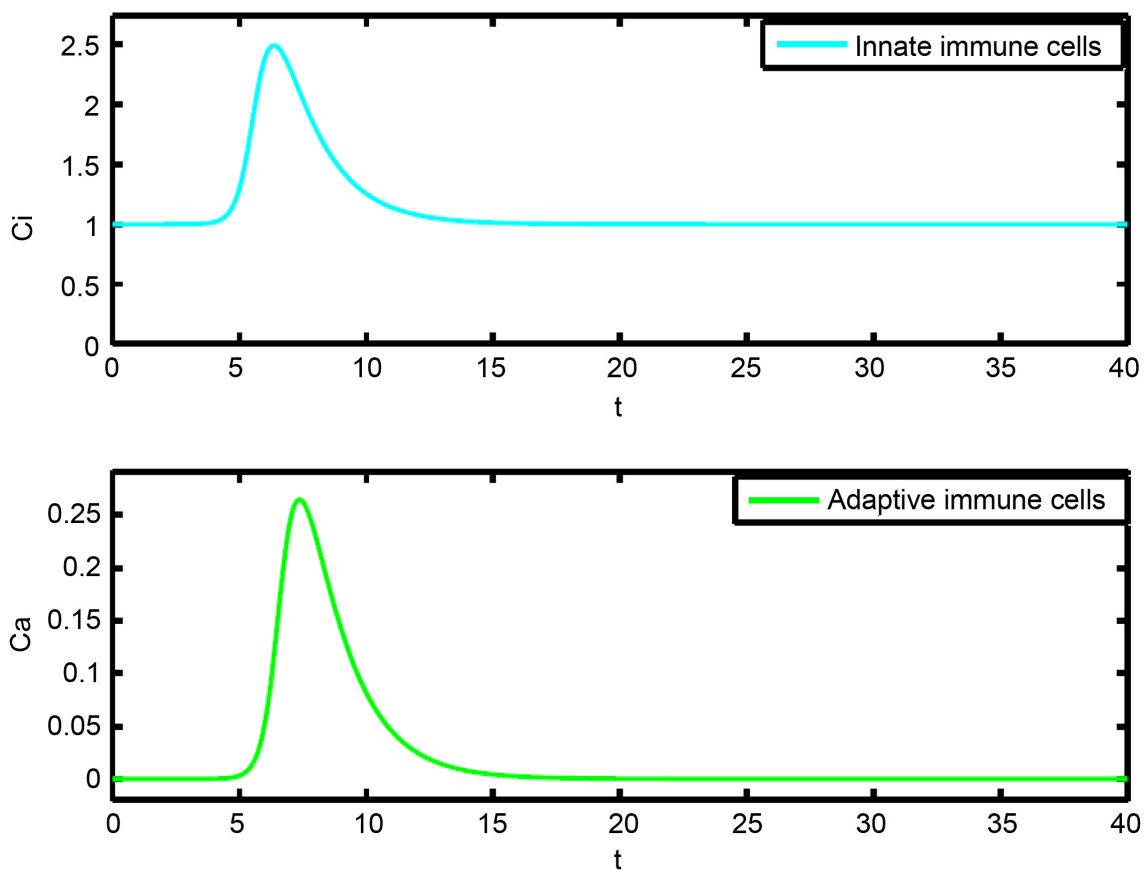

Figure 2. Results show the behavior of the concentrations of innate immune cells and the antibodies (adaptive immune cells). Both innate and adaptive immune cells decay after an initial growth.

immune cells concentration, as shown in the second graph in Figure 2. As the innate immune cells level reached its peak, there was a massive damage of gametocytes, merozoites, and infected red blood cells. As a result, the concentra- 
tions of infected red blood cells and that of merozoites died out causing no more reaction from infected red blood cells to gametocytes, see Figure 3. Hence, gametocytes concentration decayed and innate immune cells concentration restored back to normal.

When different sets of parameter values are used, the shapes of the graphs were similar, however, the minimum red blood cell levels and the times they took to reach such levels were different. This was expected because different patients react differently to disease pathology and may have different immune response statuses. For example, a healthier eating habit of an individual may promote a stronger immune system that could diminish the infection rate of red blood cells.

We also demonstrated the effect of different immune profiles on disease pathology using a death threshold defined by a third of the normal red blood cell level in Figure 4. The results suggested that both innate and adaptive immune had the minimum levels of red blood cell above the threshold level. However, in the absence of one or both of the immune types the minimum blood level was below the threshold. Similarly, the rate of production of gametocytes was higher in the absence of immunity since gametocytes are produced from infected red blood cells. The overall amount of gametocytes produced at any time $t$ from the beginning of the disease until death is illustrated in Figure 5.

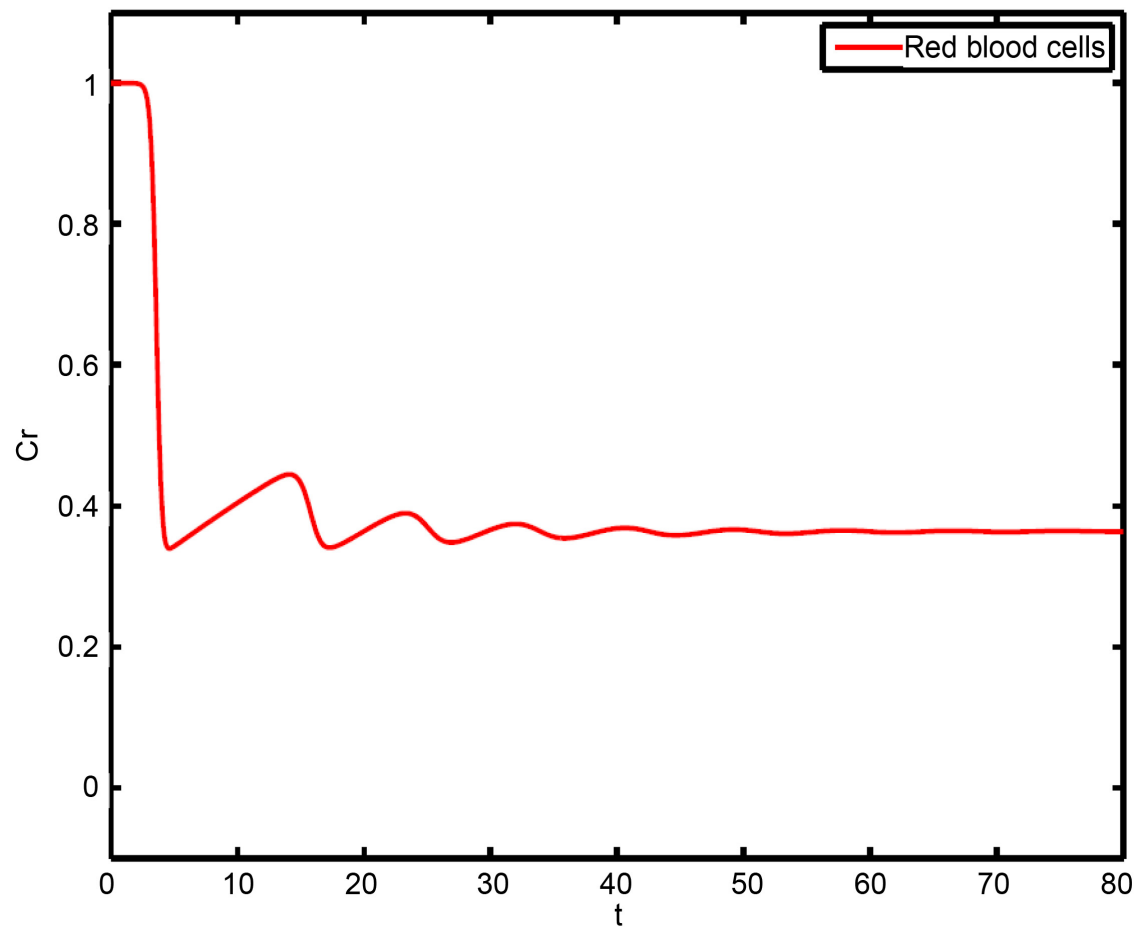

Figure 3. Result shows secondary outbreak of the malaria disease. Here, the concentration of red blood cells drops after the initial outbreak. However, recovery is initiated as the immune response gains control. The immune cells ease after the concentration of merozoites drops creating room for merozoites growth and re-invasion of the red blood cells. 


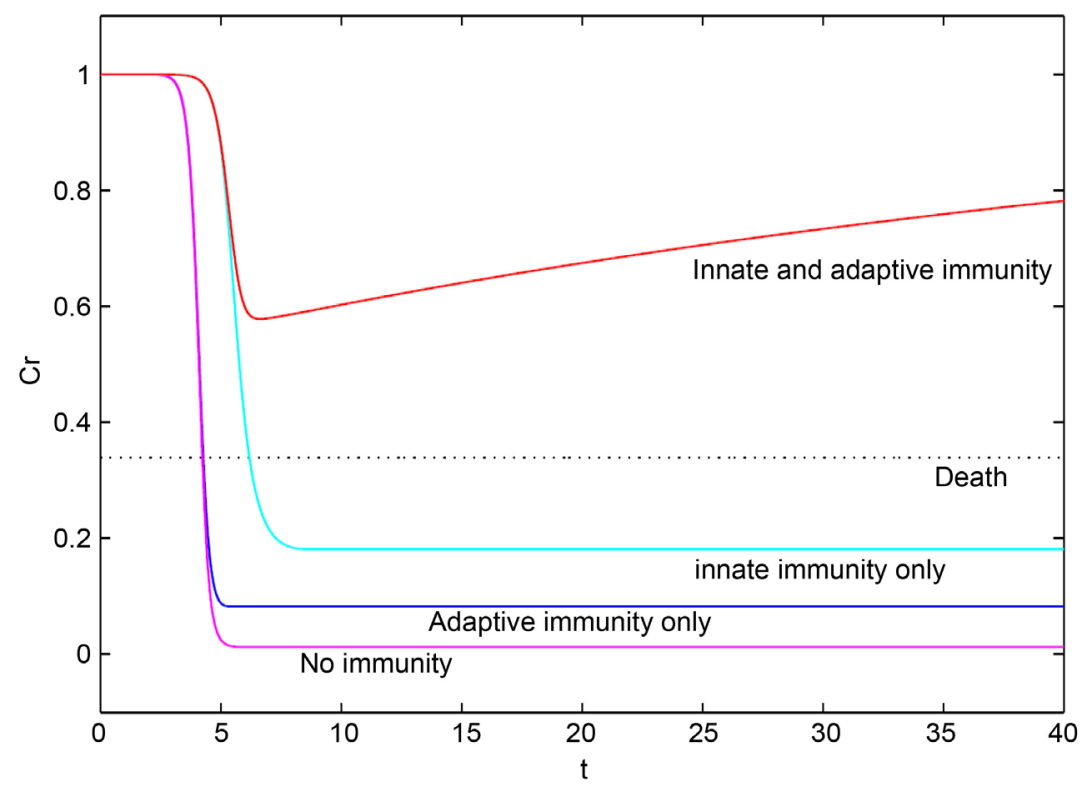

Figure 4. Results show the effect of immune response on the pathogenesis of malaria. The red curve represents concentration of red blood cells when the individual has both innate and adaptive immune response. The curves with the cyan and blue colors indicate red blood cell concentrations due to innate immunity only and adaptive immunity only, respectively. Death is assumed to occur when the red blood cell levels are below the dotted horizontal line while the magenta curve represents the red blood cell level in the absence of immunity.

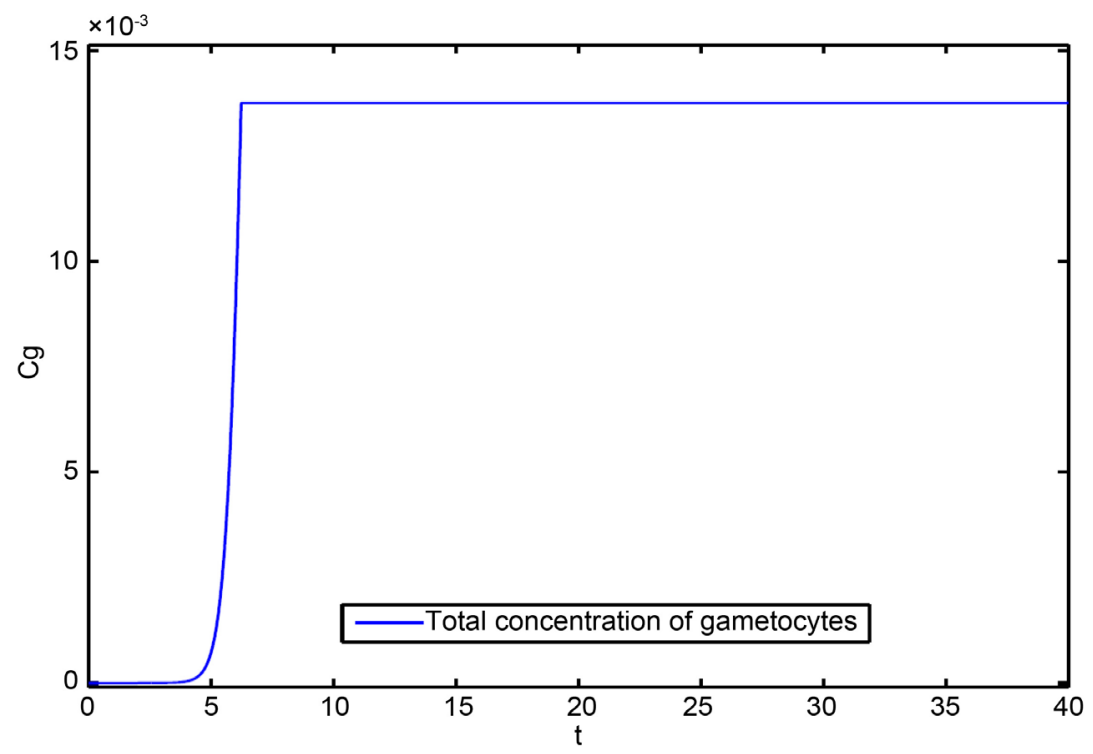

Figure 5. Results show that the total concentration of gametocyte at any point in time as the disease progresses until death is $t=6.2$.

\section{Discussion and Conclusion}

\subsection{Discussion}

We modeled in-host dynamics of malaria parasites depicting the erythrocytic blood-stage asexual parasite and gametocyte formation. The model was impor- 
tant since clinical symptoms, mortality, and disease transmission are relevant at this stage of the disease. In first contact with the parasite, the innate immune cells were activated through antigen presentation mechanism that lead to the production of antibodies. The time lagged between the parasite contact and the immune activation was signified in our simulation as $x$ and assumed to be a delay of 2 days. The simulation revealed the in-host malaria interactions with the red blood cells and the host immunity. Specifically, we observed that increased merozoites death rate decreased the concentration of infected red blood cells and increased the healthy red blood cells. Furthermore, an improved merozoites rate on susceptible red blood cells caused decrease in healthy erythrocytes. The contrary that an increased in the infected rate of healthy erythrocytes caused an increase in the density of infected red blood cells was also true. Thus, malaria control should target the infectious merozoites at the blood stage.

Although several biological factors may seem to influence this development, the primary effect appeared to be moderate or high parasite density [47]. However, the concentration of the red blood cells did not fall at once after the malaria parasite invasion. This could be partly due to recruitment of red blood cells and the intervention of innate immune cells providing mechanism to prevent the growth of infection. Another reason could be that since there were only $1.05 \times$ $10^{-7}$ initial merozoites concentration representing 35,000 merozoites, immune response was low until concentrations of infected red blood cells and merozoites reached sufficient levels. We also observed that the parasites invading the red blood cells triggered a decrease in the concentration of uninfected red blood cells, thus, increasing infected red blood cells, merozoites and gametocytes concentrations.

In addition, we observed that the severity of malaria infection increased when either the density or the activation of the innate and/or adaptive immune system was compromised. For example, a decrease in immune concentration caused erythrocytic schizogony and hence increased merozoites concentration in the host blood. Consequently, the concentrations of both the innate and adaptive immune cells surged up to reduce infected red blood cells, merozoites, and gametocytes to a small level that was inept to attack, therefore, causing the red blood cells to recover and eventually bringing the immune cells back to normal.

Considering the clinical setting case of the patient infected with malaria strain, we observed that the total amount of gametocytes at death was $6.9 \times 10^{4} \mathrm{cells} / \mu \mathrm{l}$ and it took 14 days after the release of merozoites into the blood stream for the blood level to drop to its minimum of $2.7 \times 10^{6} \mathrm{cells} / \mu \mathrm{l}$. This is within the incubation period of Plamodium falciparum malaria which is $10-15$ days. Thus, since it takes about six days for merozoites release, the likely duration from the bite to attain the minimum blood level may be about 3 weeks. Thus, the merozoites concentration peaked at $5.5 \times 10^{5} \mathrm{cells} / \mu \mathrm{l}$, which was two days prior to the red blood cells attaining its minimum level, whilst maximum adaptive immunity was achieved three days after. 
To determine the effect of the innate immune response in defending the individual against malaria, we disabled the immune response and found out that an individual without adaptive immunity was more likely to die after 18 days of parasites introduction, see Figure 4 . We observed that the horizontal line intersected with the red blood cells curve at $(6.2,0.3)$ where we have indicated death to occur at fatal red blood cells level.

\subsection{Conclusion}

In this paper, we constructed and analyzed an in-host malaria model in which the interaction between parasites, red blood cells, and immune system were formulated and examined. Mechanism of antigen presentation and time delay of innate and adaptive immune cells at the beginning of infection were established. Numerical simulations of the disease pathology from asexual erythrocyte stage to sexual exoerythrocytic stage were examined. The result revealed that intervention during malaria infection should focus on minimizing merozoite invasion rate on healthy erythrocytes and the density of merozoites in circulation. In the absence of treatment, the immune cells were vital in eliminating infected red blood cells at the blood stage. Reducing immunity propelled erythrocytic schizogony and hence increased merozoits in the host's blood and higher merozoite density increased the severity of the disease infection. However, deactivating immunity caused in fatal red blood cell count which could result to death. Therefore, the intervention could help terminate the erythrocytic schizont, leading to minimal parasite transmission of malaria. Thus, our model help to gain insights to complex dynamics and interactions of in-host malaria infection at the blood stage.

\section{Conflicts of Interest}

The author declares no conflicts of interest regarding the publication of this paper.

\section{References}

[1] WHO (2020) World Malaria Report 2020. World Health Organization, Geneva. https://www.who.int/news-room/fact-sheets/detail/malaria

[2] Kojom, L.P. and Singh, V. (2020) Prevalence of Plasmodium falciparum Field Isolates with Deletions in Histidine-Rich Protein 2 and 3 Genes in Context with Sub-Saharan Africa and India: A Systematic Review and Meta-Analysis. Malaria Journal, 19, 46. https://doi.org/10.1186/s12936-019-3090-6

[3] Soulard, V., Bosson-Vanga, H., Lorthiois, A., et al. (2015) Plasmodium falciparum Full Life Cycle and Plasmodium ovale Liver Stages in Humanized Mice. Nature Communications, 6, 7690. https://doi.org/10.1038/ncomms8690

[4] RiscoCastillo, V., Topçu, S., Marinach, C., Manzoni, G., Bigorgne, A.E., Briquet, S., Baudin, X., Lebrun, M., Dubremetz, J.F. and Silvie, O. (2015) Malaria Sporozoites Traverse Host Cells within Transient Vacuoles. Cell Host Microbe, 18, 593-603. https://doi.org/10.1016/i.chom.2015.10.006

[5] Rosenberg, R., Wirtz, R.A., Schneider, I. and Burge, R. (1990) An Estimation of the 
Number of Malaria Sporozoites Ejected by a Feeding Mosquito. Transactions of the Royal Society of Tropical Medicine and Hygiene, 84, 209-212. https://doi.org/10.1016/0035-9203(90)90258-G

[6] Spring, M., Murphy, J., Nielsen, R., Dowler, M., Bennett, J.W., Zarling, S., Williams, J., de la Vega, P., Ware, L., Komisar, J., et al. (2013) First-in-Human Evaluation of Genetically Attenuated Plasmodium falciparum Sporozoites Administered by Bite of Anopheles Mosquitoes to Adult Volunteers. Vaccine, 31, 4975-4983. https://doi.org/10.1016/j.vaccine.2013.08.007

[7] Tavares, J., Formaglio, P., Thiberge, S., Mordelet, E., VanRooijen, N., Medvinsky, A., Ménard, R. and Amino, R. (2013) Role of Host Cell Traversal by the Malaria Sporozoite during Liver Infection. Journal of Experimental Medicine, 210, 905-915. https://doi.org/10.1084/jem.20121130

[8] Vaughan, A.M., Mikolajczak, S.A., Wilson, E.M., Grompe, M., Kaushansky, A., Camargo, N., Bial, J., Ploss, A. and Kappe, S.H. (2012) Complete Plasmodium falciparum Liver-Stage Development in Liver-Chimeric Mice. Journal of Clinical Inves tigation, 122, 3618-3628. https://doi.org/10.1172/JCI62684

[9] Menendez, C., Fleming, A.L. and Alonso, P.L. (2000) Malaria-Related Anaemia. Reviews. Parasitology Today, 16, 469-476. https://doi.org/10.1016/S0169-4758(00)01774-9

[10] RegevRudzki, N., Wilson, D.W., Carvalho, T.G., Sisquella, X., Coleman, B.M., Rug, M., Bursac, D., Angrisano, F., Gee, M., Hill, A.F., et al. (2013) Cell-Cell Communication between Malaria-Infected Red Blood Cells via Exosome-Like Vesicles. Cell, 153, 1120-1133. https://doi.org/10.1016/j.cell.2013.04.029

[11] Lombardo, F. and Christophides, G.K. (2016) Novel Factors of Anopheles gambiae Haemocyte Immune Response to Plasmodium berghei Infection. Parasites \& Vectors, 9, 78. https://doi.org/10.1186/s13071-016-1359-y

[12] Crompton, P.D., Moebius, J., Portugal, S., et al. (2014) Malaria Immunity in Man and Mosquito: Insights into Unsolved Mysteries of a Deadly Infectious Disease. Annual Review of Immunology, 32, 157-187. https://doi.org/10.1146/annurev-immunol-032713-120220

[13] Clayton, A.M., Dong, Y. and Dimopoulos, G. (2014) The Anopheles Innate Immune System in the Defense against Malaria Infection. Journal of Innate Immunity, 6, 169-181. https://doi.org/10.1159/000353602

[14] Zheng, H., Tan, Z. and Xu, W. (2014) Immune Evasion Strategies of Pre-Erythrocytic Malaria Parasites. Mediators of Inflammation, 2014, Article ID: 362605. https://doi.org/10.1155/2014/362605

[15] Belachew, E.P. (2018) Immune Response and Evasion Mechanisms of Plasmodium falciparum Parasites. Journal of Immunology Research, 2018, Article ID: 6529681. https://doi.org/10.1155/2018/6529681

[16] Gomes, P.S., Bhardwaj, J., Rivera-Correa, J., Freire-De-Lima, C.G. and Morrot, A. (2016) Immune Escape Strategies of Malaria Parasites. Frontiers in Microbiology, 7, Article No. 1617. https://doi.org/10.3389/fmicb.2016.01617

[17] Bertolino, P. and Bowen, D.G. (2015) Malaria and the Liver: Immunological Hide-and-Seek or Subversion of Immunity from Within? Frontiers in Microbiology, 6, Article No. 41. https://doi.org/10.3389/fmicb.2015.00041

[18] Dennison, N.J., BenMarzouk-Hidalgo, O.J. and Dimopoulos, G. (2015) MicroRNA-Regulation of Anopheles gambiae Immunity to Plasmodium falciparum Infection and Midgut Microbiota. Developmental \& Comparative Immunology, 49, 170-178. https://doi.org/10.1016/j.dci.2014.10.016 
[19] Yam, X.Y. and Preise, P.R. (2017) Host Immune Evasion Strategies of Malaria Blood Stage Parasite. Mol. BioSyst., 13, 2498-2508.

[20] Annan, K. and Mukinay, C.D. (2017) Stability and Time-Scale Analysis of Malaria Transmission in Human-Mosquito Population. International Journal of Systems Science and Applied Mathematics, 2, 1-9. https://doi.org/10.11648/j.ijssam.20170201.11

[21] Ren, G., Zhao, X., Zhang, L., Zhang, J., L’Huillier, A., Ling, W., et al. (2010) Inflammatory Cytokine-Induced Intercellular Adhesion Molecule-1 and Vascular Cell Adhesion Molecule-1 in Mesenchymal Stem Cells Are Critical for Immunosuppression. The Journal of Immunology, 184, 2321-2328.

https://doi.org/10.4049/jimmunol.0902023

[22] Jenkins, N., Wu, Y., Chakravorty, S., Kai, O., Marsh, K. and Craig, A. (2007) Plasmodium falciparum Intercellular Adhesion Molecule-1-Based Cytoadherence-Related Signaling in Human Endothelial Cells. The Journal of Infectious Diseases, 196, 321-327. https://doi.org/10.1086/518795

[23] Mota, M.M., Jarra, W., Hirst, E., Patnaik, P.K. and Holder, A.A. (2000) Plasmodium chabaudi Infected Erythrocytes Adhere to CD36 and Bind to Microvascular Endothelial Cells in an Organ-Specific Way. Infection and Immunity, 68, 4135-4144.

https://doi.org/10.1128/IAI.68.7.4135-4144.2000

[24] Omer, F.M., Kurtzhals, J.A.L. and Riley, E.M. (2000) Maintaining the Immunological Balance in Parasitic Infections: A Role for TGF $\beta$. Parasitology Today, 16, 18-23. https://doi.org/10.1016/S0169-4758(99)01562-8

[25] Dodoo, D., Omer, F.M., Todd, J., Akanmori, B.D., Koram, K.A. and Riley, E.M. (2002) Absolute Levels and Ratios of Proinammatory and Anti-Inammatory Cytokine Production in Vitro Predict Clinical Immunity to Plasmodium falciparum Malaria. The Journal of Infectious Diseases, 185, 971-979. https://doi.org/10.1086/339408

[26] Paul, A.S., Egan, E.S. and Duraisingh, M.T. (2015) Host-Parasite Interactions That Guide Red Blood Cell Invasion by Malaria Parasites. Current Opinion in Hematology, 22, 220-226. https://doi.org/10.1097/MOH.0000000000000135

[27] Eckhoff, P. (2013) Mathematical Models of Within-Host and Transmission Dynamics to Determine Effects of Malaria Interventions in a Variety of Transmission Settings. The American Journal of Tropical Medicine and Hygiene, 88, 817-827. https://doi.org/10.4269/ajtmh.12-0007

[28] Orwa, T.O., Mbogo, R.W. and Luboobi, L.S. (2019) Multiple-Strain Malaria Infection and Its Impacts on Plasmodium falciparum Resistance to Antimalarial Therapy: A Mathematical Modelling Perspective. Computational and Mathematical Methods in Medicine, 2019, Article ID: 9783986. https://doi.org/10.1155/2019/9783986

[29] Anderson, R.M., May, R.M. and Gupta, S. (1989) Non-Linear Phenomena in Host-Parasite Interactions. Parasitology, 99, 59-79. https://doi.org/10.1017/S0031182000083426

[30] Anderson, R.M. (1998) Complex Dynamic Behaviours in the Interactions between Parasite Populations and the Host's Immune System. International Journal for Parasitology, 28, 551-566. https://doi.org/10.1016/S0020-7519(97)00207-5

[31] Hetzel, C. and Anderson, R.M. (1996) The Within-Host Cellular Dynamics of Blood Stage Malaria: Theoretical and Experimental Studies. Parasitology, 113, 25-38. https://doi.org/10.1017/S0031182000066245

[32] Gupta, S. and Anderson, R.M. (1999) Population Structure of Pathogens: The Role of Immune Selection. Parasitol. Today (Regul Ed), 15, 497-501. 
[33] Hellriegel, B. (1992) Modelling the Immune Response to Malaria with Ecological Concepts: Short-Term Behaviour against Long-Term Equilibrium. Proceedings of the Royal Society B Biological Science, 250, 249-256. https://doi.org/10.1098/rspb.1992.0156

[34] Swinton (1996) The Dynamics of Blood-Stage Malaria: Modelling Strain Specific and Strain Transcending Immunity. Models for Infectious Human Diseases. In: Isham, V. and and Medley, G., Eds., Their Structure and Relation to Data, Cambridge University Press, Cambridge, 210-212. https://doi.org/10.1017/CBO9780511662935.029

[35] Molineaux, L. and Dietz, K. (1999) Review of Intra-Host Models of Malaria. Parassitologia, 41, 221-231.

[36] White, N.J. (2004) Antimalarial Drug Resistance. Journal of Clinical Investigation, 113, 1084-1092. https://doi.org/10.1172/JCI21682

[37] Dondorp, A.M., Yeung, S., White, L., et al. (2010) Artemisinin Resistance: Current Status and Scenarios for Containment. Nature Reviews Microbiology, 8, 272-280. https://doi.org/10.1038/nrmicro2331

[38] Das, S., Hertrich, N., Perrin, A.J., WithersMartinez, C., Collins, C.R., Jones, M.L., Watermeyer, J.M., Fobes, E.T., Martin, S.R., Saibil, H.R., et al. (2015) Processing of Plasmodium falciparum Merozoite Surface Protein MSP1 Activates a Spectrin-Binding Function Enabling Parasite Egress from RBCs. Cell Host Microbe, 18, 433-444. https://doi.org/10.1016/j.chom.2015.09.007

[39] Rouzine, I.M. and McKenzie, F.E. (2003) Link between Immune Response and Parasite Synchronization in Malaria. Proceedings of the National Academy of Sciences of the United States of America, 100, 3473-3478. https://doi.org/10.1073/pnas.262796299

[40] Chiyaka, C., Garira, W. and Dube, S. (2008) Modelling Immune Response and Drug Therapy in Human Malaria Infection. Computational and Mathematical Methods in Medicine. An Interdisciplinary Journal of Mathematical, Theoretical and Clinical Aspects of Medicine, 9, 143-163. https://doi.org/10.1080/17486700701865661

[41] Tumwiine, J., Mugisha, J.Y.T. and Luboobi, L.S. (2010) A Host-Vector Model for Malaria with Infective Immigrants. J. Math. Anal. Appl., 361, 139-149.

[42] Li, Y., Ruan, S. and Xiao, D. (2011) The Within-Host Dynamics of Malaria Infection with Immune Response. Mathematical Biosciences and Engineering, 8, 999-1018. https://doi.org/10.3934/mbe.2011.8.999

[43] Tumwiine, J., Hove-Musekwa, S.D. and Nyabadza, F. (2014) A Mathematical Model for the Transmission and Spread of Drug Sensitive and Resistant Malaria Strains within a Human Population. ISRN Biomathematics, 2014, Article ID: 636973. https://doi.org/10.1155/2014/636973

[44] Tumwiine, J., Mugisha, J.Y. and Luboobi, L.S. (2008) On Global Stability of the Intra-Host Dynamics of Malaria and the Immune System. Journal of Mathematical Analysis and Applications, 341, 855-869. https://doi.org/10.1016/j.jmaa.2007.10.035

[45] Selemani, M.A., Luboobi, L.S. and Nkansah-Gyekye, Y. (2016) On Stability of the In-Human Host and In-Mosquito Dynamics of Malaria Parasite. Asian Journal of Mathematics and Applications, 2016, ama0353.

[46] Selemani, M.A., Luboobi, L.S. and Nkansah-Gyekye, Y. (2017) The In-Human Host and In-Mosquito Dynamics of Malaria Parasites with Immune Responses. New Trends in Mathematical Sciences, 5, 182-207. https://doi.org/10.20852/ntmsci.2017.196

[47] Tabo, Z., Luboobi, L.S. and Ssebuliba, J. (2017) Mathematical Modelling of the 
In-Host Dynamics of Malaria and the Effects of Treatment. Journal of Mathematics and Computer Science, 17, 1-21. https://doi.org/10.22436/jmcs.017.01.01

[48] Ngwa, G.A. and Shu, W.S. (2000) A Mathematical Model for Endemic Malaria with Variable Human and Mosquito Populations. Mathematical and Computer Modelling, 32, 747-763. https://doi.org/10.1016/S0895-7177(00)00169-2

[49] Bate, C.A.W., Taverne, J. and Playfair, J.H.L. (1988) Malarial Parasites Induce TNF Production by Macrophages. The Journal of Immunology, 64, 227-231.

[50] Elloso, M.M., Van Der Heyde, H.C., Vande Waa, J.A., Manning, D.D. and Weidanz, W.P. (1994) Inhibition of Plasmodium falciparum in Vitro by Human T Cells. The Journal of Immunology, 153, 1187-1194.

[51] Ganusov, V.V., Bergstrom, C.T. and Antia, R. (2002) Within-Host Population Dynamics and the Evolution of Microparasites in a Heterogeneous Host Population. Evolution, 56, 213-223. https://doi.org/10.1111/j.0014-3820.2002.tb01332.x

[52] McQueen, P.G. and McKenzie, F.E. (2008) Host Control of Malaria Infections: Constraints on Immune and Erythropoeitic Response Kinetics. PLoS Computational Biology, 4, Article No. e1000149. 